\title{
Dummy molecularly imprinted polymers based on a green synthesis strategy for magnetic solid-phase extraction of acrylamide in food samples
}

\author{
Ahmad Reza Bagheri ${ }^{\mathrm{a}}$, Maryam Arabi $^{\mathrm{a}}$, Mehrorang Ghaedi ${ }^{\mathrm{a}, *}$, Abbas Ostovan $^{\mathrm{a}}$, Xiaoyan Wang ${ }^{\mathrm{b}, \mathrm{c}}$, \\ Jinhua Li ${ }^{\mathrm{b}}$, Lingxin Chen ${ }^{\mathrm{b}, \mathrm{d}, * * *}$ \\ ${ }^{a}$ Chemistry Department, Yasouj University, Yasouj 75918-74831, Iran \\ ${ }^{\mathrm{b}}$ CAS Key Laboratory of Coastal Environmental Processes and Ecological Remediation, Research Center for Coastal Environmental Engineering Technology of Shandong \\ Province, Yantai Institute of Coastal Zone Research, Chinese Academy of Sciences, Yantai 264003, China \\ ${ }^{\mathrm{c}}$ School of Pharmacy, Binzhou Medical University, Yantai 264003, China \\ ${ }^{\mathrm{d}}$ College of Chemistry and Chemical Engineering, Qufu Normal University, Qufu 273165, China
}

\section{A R T I C L E I N F O}

\section{Keywords:}

Molecularly imprinted polymers

Dummy template

Green synthesis

Magnetic solid-phase extraction

Experimental design

Acrylamide

\begin{abstract}
A B S T R A C T
In this work, novel dummy molecularly imprinted polymers (DMIP) with propanamide as a dummy template molecule were prepared based on a green synthesis strategy of less consumption of hazardous/organic reagents and at mild conditions for magnetic solid-phase extraction (MSPE) of acrylamide in biscuit samples, followed by high performance liquid chromatography (HPLC) determination. The resultant DMIP was well characterized by FT-IR, SEM, TEM and VSM, exhibiting uniform nanoscale coreshell structure and good magnetic property in favor of simple rapid separation. Several main variables influencing MSPE efficiency were investigated, including DMIP dosage, sample solution $\mathrm{pH}$, extraction time and desorption solvent; central composite design (CCD) and response surface methodology (RSM) were employed to assist in the MSPE condition optimization with rapidity and reliability. Under optimized conditions, excellent linearity for acrylamide was obtained in the

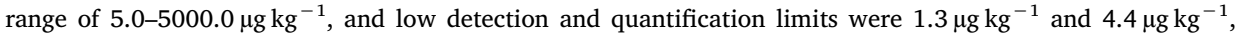
respectively. The method recoveries at five spiked concentrations were found within $86.0-98.3 \%$ with relative standard deviations (RSDs) of 1.2-4.1\%. Furthermore, endogenous acrylamide was detected in five different biscuit samples and the RSDs values were lower than 3.3\%. The present study suggested promising perspectives of water-compatible eco-friendly DMIP based MSPE-HPLC method for highly effective sample pretreatment and targeted analytes determination in complicated matrices.
\end{abstract}

\section{Introduction}

Acrylamide also named 2-propenamide, as a small unsaturated hydrophilic amide, has been widely used in water treatment, food packaging, plastic products, paper, cosmetics, and cement production [1]. In 1994, International Agency for Research on Cancer (IARC) identified acrylamide as neurotoxic compound and "probably carcinogenic to humans" (Group 2A) which could be readily absorbed through the skin (IARC, 1994). A few years later in 2002, Stockholm University and Swedish National Food Administration reported that heat treat processed foods viz. French fries, breakfast cereals and coffee contained large amounts of acrylamide [2]. Acrylamide can be easily formed by Maillard reaction during frying baking and grilling carbohydrate-rich food such as potato chips, crispy layer rice (Iranian traditional food) and biscuit, when they are exposed to high temperature exceeding $120^{\circ} \mathrm{C}$ under low moisture conditions $[3,4]$. According to the World Health Organization (WHO) guideline, the threshold limit for acrylamide in drinking water is $0.5 \mu \mathrm{g} \mathrm{L}^{-1}$ [5]. It should be pointed out that an exposure to low amount of acrylamide $\left(0.5-0.98 \mu \mathrm{g} \mathrm{kg}^{-1}\right.$ b.w./day for adults $>15$ years and $1.25-2.54 \mu \mathrm{gg}^{-1}$ b.w./day for 3-14 years) by consuming of foodstuffs increases the risk of cancer [6]. Therefore, accurate determination of acrylamide in food stuffs is a vital issue for food safety and human health.

Recently, chromatographic techniques coupled with sensitive detectors (hyphenated techniques) such as gas chromatography-mass spectrometry (GC-MS) [7], GC-electron capture spectrometry [8],

\footnotetext{
* Corresponding author.

** Corresponding author at: CAS Key Laboratory of Coastal Environmental Processes and Ecological Remediation, Research Center for Coastal Environmental Engineering Technology of Shandong Province, Yantai Institute of Coastal Zone Research, Chinese Academy of Sciences, Yantai 264003, China.

E-mail addresses: m_ghaedi@mail.yu.ac.ir (M. Ghaedi), 1xchen@yic.ac.cn (L. Chen).
} 
liquid chromatography-MS (LC-MS) [9] and LC-MS/MS [10] have been employed as powerful tools for monitoring and quantitative analysis of acrylamide in different complex food. Nevertheless, direct injection of raw extracts easily leads to poor separation and pollutes separator/ detector due to the complexity of sample's matrices and the low concentration of target analytes [11]. Hence, for accurate quantification of trace acrylamide in complicated food matrices, efficacious sample preparation and instrumental analysis are equally important. Among various sample preparation techniques, great attention has been paid to magnetic solid-phase extraction (MSPE) owing to unique superiorities viz. least consumption of organic solvent, no need to conditioning the sorbent and also column packing elimination, and high contact surface enhancing efficiency and repeatability, and on the other part, magnetic sorbents are gathered easily by magnetic field, omitting filtration, centrifugation and precipitation procedures $[12,13]$. Despite foresaid advantages, MSPE often suffers from poor selectivity which could be overcome by combination with selective adsorbents.

Molecularly imprinted polymers (MIPs), based on creation of specific recognition sites in strict polymer network that have complementary shape, size and functional groups toward the imprinted molecule, have attracted increasing interest as selective adsorbents for isolation and enrichment of small organic molecules, metal ions, biomacromolecules, etc [14]. Meanwhile, MIPs face to some major drawbacks [14], for example, especially while acrylamide used as template during traditional MIPs preparation and subsequently the synthesized MIPs applied as sorbent during extraction process. Firstly, acrylamide is high toxicity and strong linking through double bond $(\mathrm{C}=\mathrm{C})$ with crosslinker (in radical polymerization) and could act as a functional monomer which cause failure to be removed after exhaustively eluting [15]. Moreover, template molecules are embedded too deep in threedimensional polymer structure and easily cause inadequate removal of template, so available sites are dramatically reduced. On the other hand, undesirable mass-transfer and binding capacity have serious impact on the accuracy of quantitative analysis $[16,17]$. To address this obstacle, we purposed to employ dummy imprinting strategy for acrylamide recognition, by selecting propanamide as dummy template owing to its similarity of shape, size, and functional groups without interference in analytical determination of acrylamide. By using dummy imprinting strategy not only the leaching of residual template can be avoided easily that would enhance accuracy of analytical method, but also it would lead to increasing the number of specific binding sites and adsorption capacity.

On the other hand, it has been largely proved that, MIPs often fail to show high selectivity and rebinding ability toward small organic molecules in aqueous media. The shrinking/swelling of the MIPs, namely specific cavities are easily resized in aqueous media, significantly decreases their practical applications in such areas as environmental and food analysis $[18,19]$. Meanwhile, in majority of MIPs synthesis routes such as precipitation [20], sol-gel [21], and emulsion [22], amounts of organic porogens and/or toxic reagents are usually used, which is not economic or user-friendly and is in contrast with principles of green chemistry. To overcome the two challenges, aqueous-phase synthesis is highly desirable.

Therefore, in this work, a novel green synthesis strategy was proposed for preparation of dummy MIP (DMIP) in aqueous media without consuming organic solvents, which were subsequently used as sorbents of MSPE for recognition and extraction of acrylamide in biscuit samples, followed by HPLC determination. Chitosan as versatile natural hydrophilic polymer in presence of dummy template was employed to construct three-dimensional imprinted network in aqueous media. The particular synthesis route is presented by following steps in sequence: (I) preparation of $\mathrm{Fe}_{3} \mathrm{O}_{4}$ nanoparticles as an appropriate support for the surface imprinting due to its magnetic property, high surface area, suitable functionality for further modification, and biocompatibility, non-toxicity and eco-friendliness. (II) encapsulation of $\mathrm{Fe}_{3} \mathrm{O}_{4}$ nanoparticles by anchoring hydrophilic polyethylene glycol chain by facile and one step process with no consumption of organic solvent; and (III) polymerization and imprinting of chitosan on the surface of modified $\mathrm{Fe}_{3} \mathrm{O}_{4}$ nanoparticles to produce specific molecular binding sites. The asprepared water-soluble DMIP was used as MSPE sorbent for efficient clean-up and preconcentration of acrylamide, and the MSPE conditions were optimized with the aid of experimental design of central composite design (CCD) and response surface methodology (RSM) with minimum effort, expense, and higher accuracy. The DMIP based MSPE method was well validated and successfully applied to biscuit sample analysis, demonstrating high sensitivity, repeatability, simplicity, rapidity, good practical feasibility, and environmental benignity.

\section{Experimental}

\subsection{Materials and instruments}

Iron (III) chloride hexahydrate $\left(\mathrm{FeCl}_{3} \cdot 6 \mathrm{H}_{2} \mathrm{O}\right)$, iron (II) chloride tetrahydrate $\left(\mathrm{FeCl}_{2} \cdot 4 \mathrm{H}_{2} \mathrm{O}\right)$, poly(ethylene glycol) (PEG) with average W\% of $6000 \mathrm{~g} \mathrm{~mol}^{-1}$, acrylamide, propanamide, sodium hydroxide $(\mathrm{NaOH})$, methanol, ethanol, acetonitrile (HPLC grade), acetic acid, and ammonium hydroxide $\left(\mathrm{NH}_{4} \mathrm{OH}\right)$ were purchased from Merck (Darmstadt, Germany). Chitosan powder (low molecular weight), with $\geq 75.0 \%$ degree of deacetylation and 20-30 caps of viscosity, was supplied from Sigma-Aldrich (St. Louis, MO, USA). Ultrapure water was obtained from a Milli-Q water purification system (Millipore, Bedford, MA, USA).

Scanning electron microscope (SEM) images were recorded on a Hitachi S4160 microscope and transmission electron microscopy (TEM) images on a Hitachi S-570 microscope, from Hitachi (Tokyo, Japan). Magnetic properties of materials were studied using a vibrating sample magnetometer (VSM, LDJ 9600-1, USA). For identification of functional groups Fourier transform-infrared spectroscopy (FT-IR-8300, Shimadzu) using $\mathrm{KBr}$ pellet was employed. Tecno-GAZ SPA Ultra Sonic System at $40 \mathrm{kHz}$ coupled to a temperature controller was used for adsorption/desorption processes.

Acrylamide determination was performed by an Agilent 1100 liquid chromatography (Wilmington, DE, USA) equipped with a micro vacuum degasser (model G1379A), a quaternary pump (model G1311A), a multiple wavelength detector (model G13658), a sample injection valve with a $20 \mu \mathrm{L}$ sample loop, and an Agilent $\mathrm{C}_{18}$ column (4.6 mm i.d. $250 \mathrm{~mm}, 5 \mu \mathrm{m})$. Mobile phase consisted of a water-methanol (95:5, v/v) which was filtered through a $0.45 \mu \mathrm{m}$ filter, degassed under vacuum and passed with the flow rate of $1.0 \mathrm{~mL} \mathrm{~min}^{-1}$. Separation process was carried out at ambient temperature and detector wavelength was set at $210 \mathrm{~nm}$.

\subsection{Preparation of DMIP}

First, $\mathrm{Fe}_{3} \mathrm{O}_{4}$ nanoparticles were synthesized using chemical co-precipitation according to our previous work [23]. Briefly, $15 \mathrm{mmol}$ of $\mathrm{FeCl}_{3} \cdot 6 \mathrm{H}_{2} \mathrm{O}$ and $10 \mathrm{mmol}$ of $\mathrm{FeCl}_{2} \cdot 4 \mathrm{H}_{2} \mathrm{O}$ were added to $80 \mathrm{~mL}$ of deionized water in a $250 \mathrm{~mL}$ tri-neck flask under nitrogen atmosphere. $50 \mathrm{~mL}$ ammonium solution was added dropwise into the flask under vigorous stirring of nitrogen stream to get a black mixture. Subsequently, the black mixture was strongly stirred and heated at $80^{\circ} \mathrm{C}$ for $30 \mathrm{~min}$. Then, the black precipitate was separated by Nd-Fe-B permanent magnet and repeatedly rinsed with deionized water until achieving a neutral $\mathrm{pH}$ for the washed solution and dried under vacuum at $60^{\circ} \mathrm{C}$

Surface modification of $\mathrm{Fe}_{3} \mathrm{O}_{4}$ nanoparticles was performed by onepot, green, and very quick method with PEG as surface modifier. Briefly, $10 \mathrm{~g}$ of PEG was dissolved in $30 \mathrm{~mL}$ of deionized water and $2.0 \mathrm{~g}$ $\mathrm{Fe}_{3} \mathrm{O}_{4}$ nanoparticles were whisked into solution. After sonicating for 30 min, PEG- $\mathrm{Fe}_{3} \mathrm{O}_{4}$ particles were separated by external magnetic field, washed with deionized water, and dried at $60^{\circ} \mathrm{C}$ for $12 \mathrm{~h}$.

The basic preparation procedure for DMIP is schematically shown in Fig. $1.0 .5 \mathrm{~mL}$ acetic acid was added into $50 \mathrm{~mL}$ of deionized water 


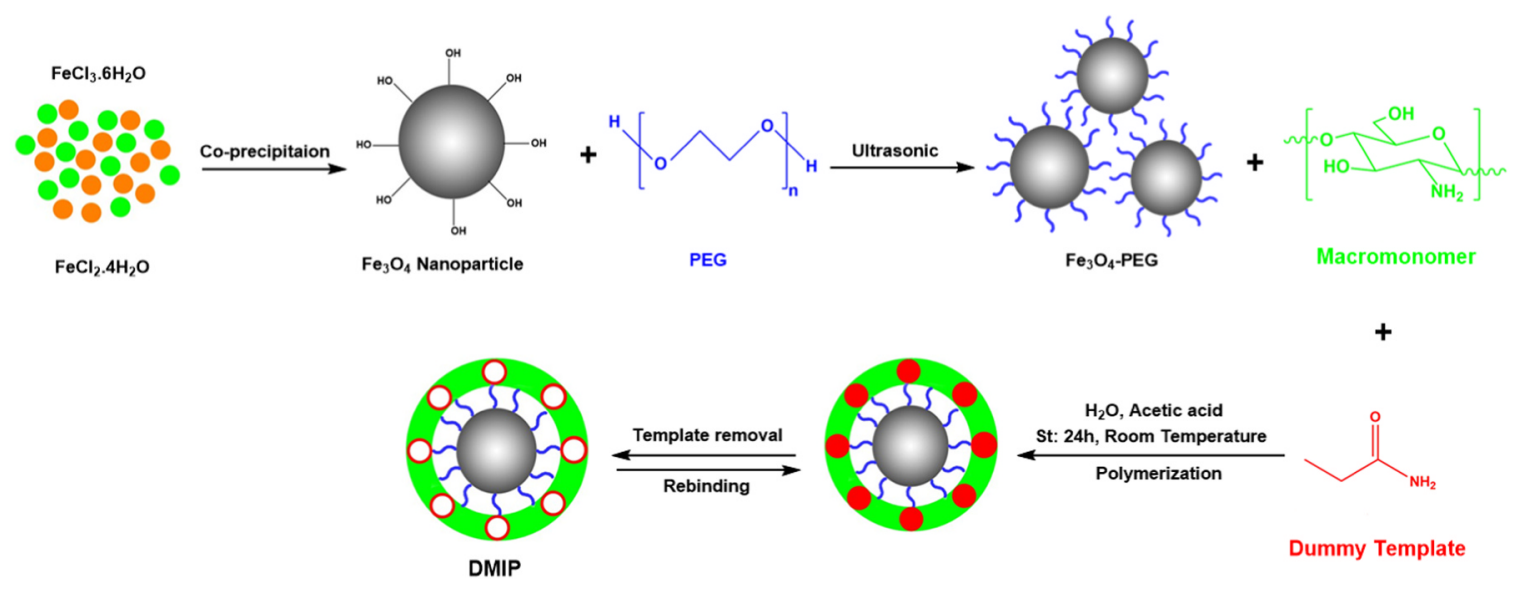

Fig. 1. The basic preparation procedure of DMIP.

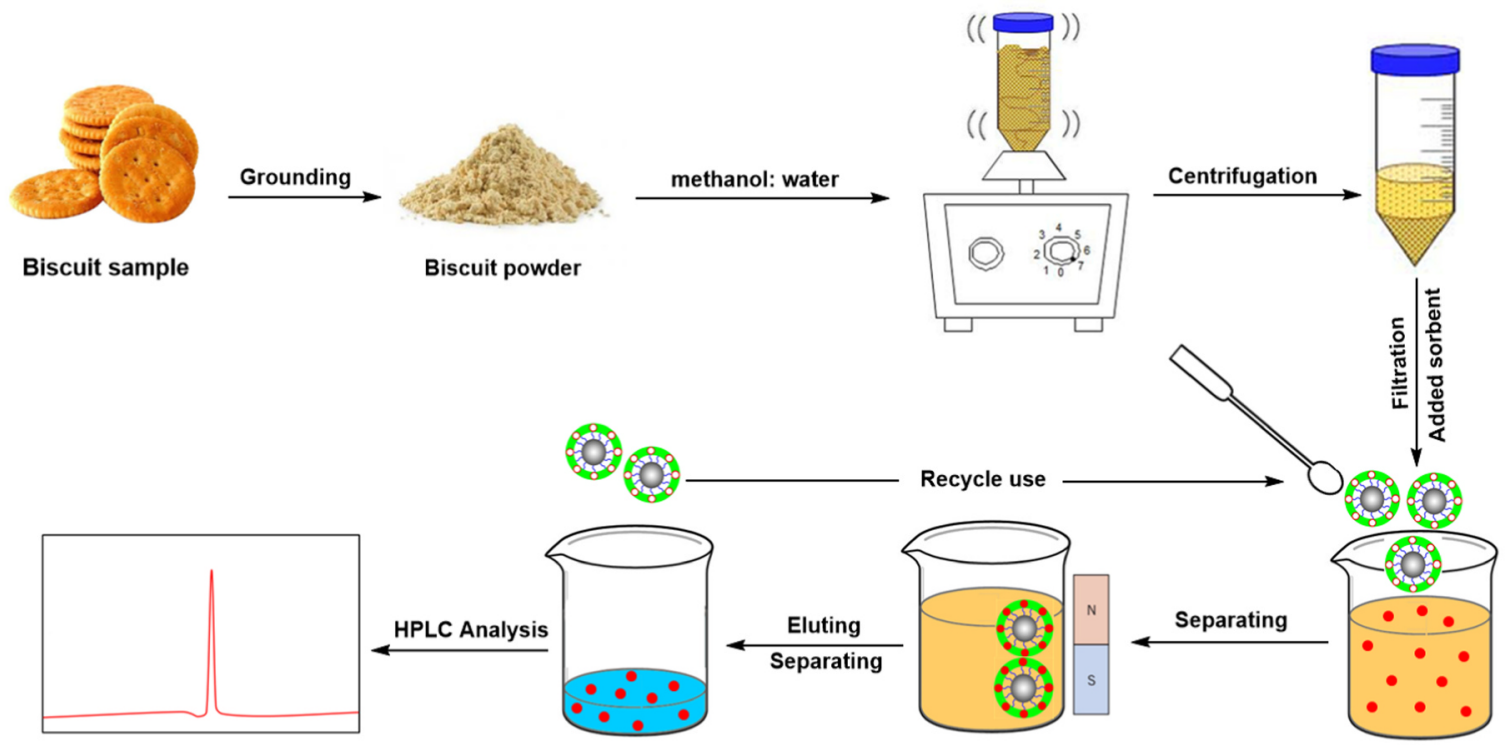

DMIP

- Acrylamide

Fig. 2. The DMIP based MSPE procedure for acrylamide extraction.

under stirring, followed by dissolving $2 \mathrm{mmol}$ of propanamide (dummy template). Then, $0.52 \mathrm{~g}$ chitosan was added to the mixture, kept stirring to appearance clear solution and allowing forming functional monomer-dummy template preassembly for $12 \mathrm{~h}$. Next, $0.25 \mathrm{~g}$ PEG$\mathrm{Fe}_{3} \mathrm{O}_{4}$ was added to above solution followed by stirring vigorously and polymerization was allowed to proceed for $24 \mathrm{~h}$ at ambient temperature. Afterward, $50 \mathrm{~mL}$ of $1 \mathrm{~mol} \mathrm{~L}^{-1} \mathrm{NaOH}$ solution was transferred swiftly into the resultant solution and kept stirring for $3 \mathrm{~h}$. Then, the prepared material was collected by external magnetic field followed by washing with deionized water repeatedly until reaching natural $\mathrm{pH}$ and was re-dispersed in $0.5 \mathrm{~mol} \mathrm{~L}^{-1} \mathrm{H}_{2} \mathrm{SO}_{4}$ for 5 min under ultrasonic irradiation. Finally, the polymer material was washed repeatedly with dispersion in the eluent of methanol-deionized water (50:50, v/v), due to the high solubility of dummy template in the eluent, and then was easily rapidly isolated with external magnetic field, until the imprinted molecule propanamide could not be detected anymore by HPLC-UV in concentrated eluent. Consequently, DMIP was obtained. The non-imprinted polymers (NIP) were synthesized using the same procedure but without the addition of dummy template.

\subsection{Adsorption test}

To investigate the binding capacity of acrylamide by the prepared sorbents, $30 \mathrm{mg}$ of DMIP or NIP was suspended into $15 \mathrm{~mL}$ of aqueous solution of acrylamide ( $\mathrm{pH}$ adjusted to 5.0) at varied initial concentrations (50.0-600.0 $\mathrm{mg} \mathrm{L}^{-1}$ ). The mixture was mechanically stirred at $25{ }^{\circ} \mathrm{C}$ for $20 \mathrm{~min}$, and then the sorbents were collected by an external magnetic field, and residual (non-sorbed) concentrations of acrylamide were determined by HPLC. The amount of acrylamide adsorbed by the DMIP/NIP was calculated according to the following equation:

$Q=\left(C_{0}-C_{t}\right) V / m$

where $Q\left(\mathrm{mg} \mathrm{g}^{-1}\right)$ is the sorption amount of acrylamide; $C_{0}\left(\mathrm{mg} \mathrm{mL}^{-1}\right)$ is the initial concentration of acrylamide, $C_{\mathrm{t}}\left(\mathrm{mg} \mathrm{mL}^{-1}\right)$ is the supernatant concentration of acrylamide after adsorption; $V(\mathrm{~mL})$ is the total volume of sample solution and $m$ is the mass of the sorbent used.

As well as, the binding kinetics of DMIP and NIP were analyzed at different time period ranging from 1 to 28 min following addition of $40 \mathrm{mg}$ of DMIP/NIP to $15 \mathrm{~mL}$ aqueous solution of acrylamide 


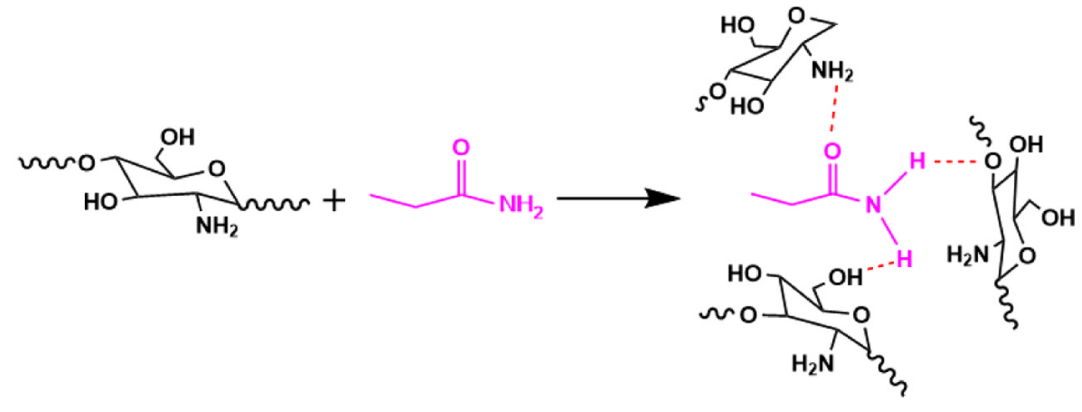

Prepolymer Complex

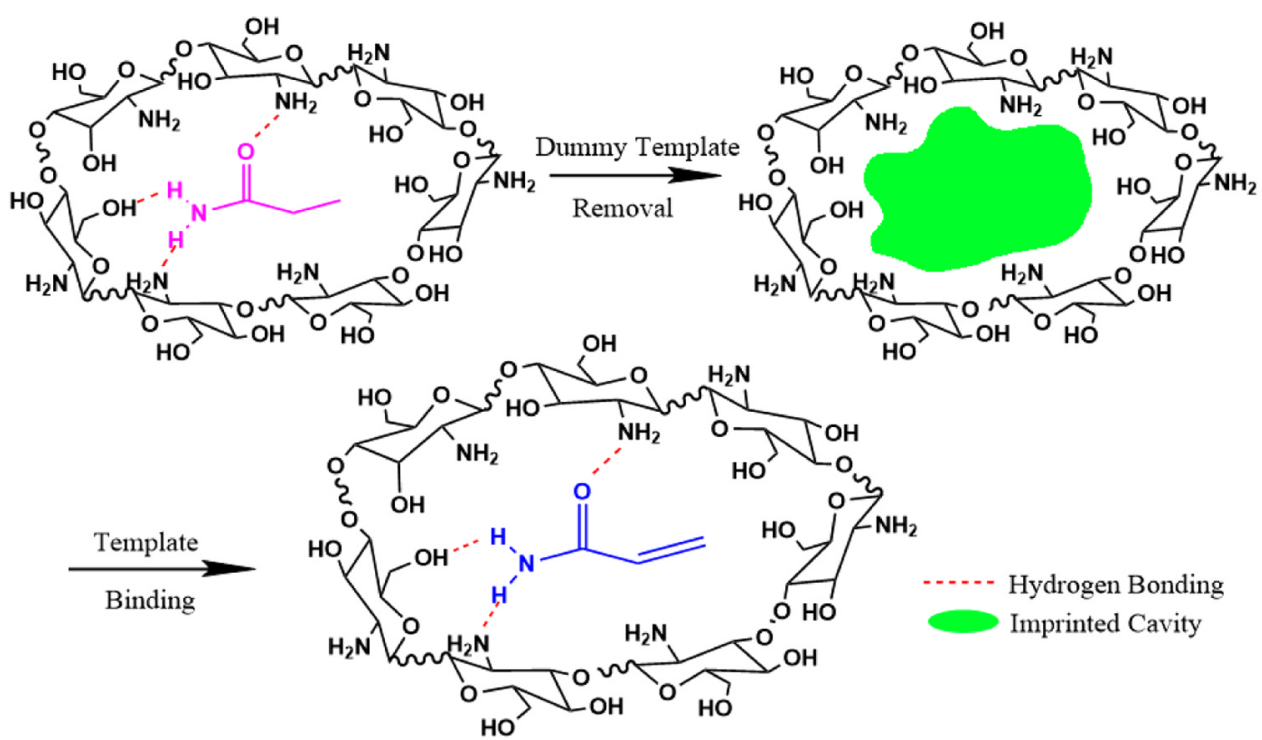

Fig. 3. Schematic for possible hydrogen bonding formation.

(450.0 $\mathrm{mg} \mathrm{L}^{-1}$ ). Then, the sorbents were isolated and the residual acrylamide in supernatant solution were determined by HPLC.

\subsection{Preparation of calibration standard and MSPE procedure of acrylamide from biscuit samples}

A standard stock solution of acrylamide was prepared in deionized water at $1.0 \mathrm{mg} \mathrm{mL}^{-1}$ and kept in $4^{\circ} \mathrm{C}$. Subsequently, the working solutions were obtained by the appropriate level of dilution with deionized water. Five different brands of biscuit samples were purchased from local supermarket in Yasouj and stored at $4{ }^{\circ} \mathrm{C}$ in dark before use. Fig. 2 displays the DMIP based MSPE procedure. Biscuit samples were grounded and blended into the homogenous powder by a mortar and pestle. Then, $2.0 \mathrm{~g}$ biscuit powder mixed with $15 \mathrm{~mL}$ methanol:water (50:50 v/v) was extracted by vigorous shaking for $30 \mathrm{~min}$. Three different solvents including methanol, deionized water, and methanol/ deionized water $(50: 50, v / v)$, were investigated for extraction of acrylamide. Experimental results suggested methanol/deionized water (50:50, v/v) provided the maximum extraction efficiency due to polarity and miscibility of acrylamide in the mixed polar solvents. Afterwards, the extract was held in an ice bath for $30 \mathrm{~min}$ to create two phases followed by centrifugation and filtration to obtain clear extract solution. At the next, $40 \mathrm{mg}$ of DMIP was added into the extract solution with $\mathrm{pH}$ adjusted at 5.0 followed by ultrasonication for $20 \mathrm{~min}$ at ambient temperature. The DMIP was separated by a magnet from the solution, and subsequently acrylamide was eluted by re-dispersion of DMIP in $2 \mathrm{~mL}$ of methanol/ammonium hydroxide (90:10, v/v) by sonication. The eluent was evaporated to dryness under nitrogen stream in water bath $\left(35^{\circ} \mathrm{C}\right)$ and the residue was subsequently reconstituted in
$50 \mu \mathrm{L}$ mobile phase (deionized water/methanol (95:5, v/v)) and ready for HPLC-UV analysis.

\subsection{Building of calibration curves in standard aqueous solution and biscuit samples}

Calibration curves were built in standard aqueous solution and biscuit samples, respectively. In this regard, blank aqueous solutions and biscuit samples were spiked with different concentrations of acrylamide, and the acrylamide was extracted under the optimized conditions and analyzed according to the method described in Section 2.4.

\section{Results and discussion}

\subsection{Preparation of DMIP}

Fig. 1 illustrates the schematic preparation procedure of DMIP. First, $\mathrm{Fe}_{3} \mathrm{O}_{4}$ nanoparticles were synthesized by coprecipitation method. The criterion for selection of coprecipitation method is based on less consumption of hazardous chemicals and organic solvents, although magnetic nanoparticles synthesized by a solvothermal method have higher magnetic response [24]. Furthermore, $\mathrm{Fe}_{3} \mathrm{O}_{4}$ nanoparticles are highly compatible with aqueous phase and can be decorated with hydroxyl groups which offer excellent sites for further interfacial bond and interaction between the $\mathrm{Fe}_{3} \mathrm{O}_{4}$ and PEG. Prior to polymerization, $\mathrm{Fe}_{3} \mathrm{O}_{4}$ was functionalized with PEG chain in very short time and at ambient temperature. In order to improve the stability of $\mathrm{Fe}_{3} \mathrm{O}_{4}$, various surface modifiers have been applied for encapsulation of $\mathrm{Fe}_{3} \mathrm{O}_{4}$ viz. $\mathrm{SiO}_{2}$, carbon, oleic acid, chitosan, and PEG. The period of encapsulation 
was dramatically shortened by treating with PEG which was 12 times lower than mentioned other surface modifiers. Therefore, we adopted PEG for surface modification which have several merits including: 1) PEG is hydrophilic, cheap, bio-compatible and environmentally friendly $[25]$, 2) surface modification was quickly performed in one pot in aqueous media at ambient temperature, 3) $\mathrm{Fe}_{3} \mathrm{O}_{4}$ with slight acidic properties in aqueous media [26] is protonated easily that can electrostatically interact with the hydroxyl groups of PEG [27]. Moreover, hydrogen bonds between hydroxyl groups of $\mathrm{Fe}_{3} \mathrm{O}_{4}$ and hydroxyl groups of PEG can form that leads to impressive capsulation [28]. Next, a layer of chitosan network was anchored onto $\mathrm{Fe}_{3} \mathrm{O}_{4} @$ @EG via one-pot facile surface imprinting in the presence of propanamide as dummy template. Chitosan could significantly interact with PEG chain with strong electrostatic interactions in acidic media whereas chitosan was protonated and PEG induced negative charge. Chitosan was selected as natural functional monomer that could supply four supreme benefits: 1) chitosan is non-toxic, cheap, readily available, biocompatible, biodegradable and easy to polymerize in mild condition; 2) because of multifunctional groups (-OH, $\left.-\mathrm{NH}_{2},-\mathrm{O}-\right)$ in its structure, it could significantly interact with propanamide in aqueous media with at least byeffects of water to form sustainable hydrogen bonding and preassembled complex, as shown in Fig. 3, herein, chitosan was used as monomer, which effectively resolved radical polymerization problems owing to using traditional monomers etc; 3 ) due to the similarity between the hydrophilicity of chitosan and acrylamide, it is a good idea to convert chitosan to specific imprinted network; 4) it could simplify cross-linking by sulfuric acid with ionic reaction very quickly in mild conditions [29] that would minimize swelling of chitosan avoiding resizing/deformation of specific cavities and improving the mechanical stability. Although, enhancing MIP performance is a vital task, observing the principles of green chemistry is equally important. Therefore, the combination of molecular imprinting technology with green synthesis approach is promising for separation of small organic molecules.

The synthesis procedure was compared with that reported in terms of precursors and synthesis conditions [15,30-34]. As can be seen from Table S1, in all the reported synthesis routes, chemical precursors and organic solvents are used which are hazardous and expensive. In addition, temperature controlling is another disadvantage. Interestingly, our proposed synthesis procedure benefits from remarkable superiorities viz. eco-friendliness, cost-effectiveness, simplicity, rapidity and high-efficiency.

\subsection{Characterization of DMIP}

The prepared DMIP was characterized by FT-IR, SEM/TEM and VSM. Fig. 4 shows the FT-IR spectra of $\mathrm{Fe}_{3} \mathrm{O}_{4}$ (a), $\mathrm{Fe}_{3} \mathrm{O}_{4}$ @PEG (b), propanamide-contained DMIP (c) and propanamide-removed DMIP (d). The strong band at $570 \mathrm{~cm}^{-1}$ in all samples was clearly observed that was attributed $\mathrm{Fe}-\mathrm{O}$ groups. Peak intensity of $\mathrm{Fe}-\mathrm{O}$ in DMIP was lower than $\mathrm{Fe}_{3} \mathrm{O}_{4} @$ @EG and $\mathrm{Fe}_{3} \mathrm{O}_{4}$ which confirmed $\mathrm{Fe}_{3} \mathrm{O}_{4}$ was decorated with PEG and MIP layer. The broad absorption peak around $3445 \mathrm{~cm}^{-1}$ corresponded to stretching vibrations of $\mathrm{O}-\mathrm{H}$ groups of PEG (Fig. 4b). The C-O symmetric stretching observed at $1107 \mathrm{~cm}^{-1}$ demonstrated that the PEG was successfully covered onto the surfaces of $\mathrm{Fe}_{3} \mathrm{O}_{4}$ nanoparticles. The characteristic peaks of DMIP at $1630 \mathrm{~cm}^{-1}$ and $1154 \mathrm{~cm}^{-1}$ could be attributed to the bending vibration of $\mathrm{NH}_{2}$ and $\mathrm{C}-\mathrm{N}$ stretching vibration, respectively, which proved that chitosan layer was anchored onto the $\mathrm{Fe}_{3} \mathrm{O}_{4} @$ PEG. Moreover, the disappearance of $\mathrm{C}=\mathrm{O}$ band at $1700 \mathrm{~cm}^{-1}$ (Fig. 4d) proved that dummy template has been removed from the DMIP.

Surface morphology and particle size of DMIP were investigated by SEM and TEM. SEM image indicated that DMIP had regular spherical shape, and relatively identical size distribution with no significant aggregation (Fig. S1). As seen, DMIP had velvety surface which might be related to the removal of dummy template and solvent molecules that were trapped in polymer network. It should be noticed that DMIP had nanoscale diameter (below $100 \mathrm{~nm}$ ), which would provide supreme structure to facilitate mass transfer of propanamide, and after dummy template removal, would cause high adsorption capacity, accessible specific cavities and fast sorption kinetics. Meanwhile, as can be seen from TEM image (Fig. S2), there were dark regions suggesting that $\mathrm{Fe}_{3} \mathrm{O}_{4}$ nanoparticles were embedded into PEG and chitosan substrate. Although the FT-IR spectra demonstrated successful synthesis of imprinted chitosan on the $\mathrm{Fe}_{3} \mathrm{O}_{4} @$ @PEG, PEG and chitosan network couldn't be seen clearly in TEM image; possibly, the density of PEG and chitosan was not much to be seen like $\mathrm{Fe}_{3} \mathrm{O}_{4}$ nanoparticles [35]. Bare $\mathrm{Fe}_{3} \mathrm{O}_{4}$ nanoparticles were aggregated owing to magnetic feature of $\mathrm{Fe}_{3} \mathrm{O}_{4}$ nanoparticles [36]. After polymerization of chitosan on the surface of $\mathrm{Fe}_{3} \mathrm{O}_{4}$ nanoparticles, the DMIP had good dispersibility due to the enhancement of repulsive force among the nanoparticles [37]. In addition, thickness of imprinted shell layer could ameliorate the mass transfer rate for swift adsorption/desorption of analyte. These results implied that DMIP nanospheres were successfully synthesized.

As for hysteresis loops of $\mathrm{Fe}_{3} \mathrm{O}_{4}$ and DMIP from the magnetization curve by VSM test, as shown Fig. S3, both samples hysteresis showed a

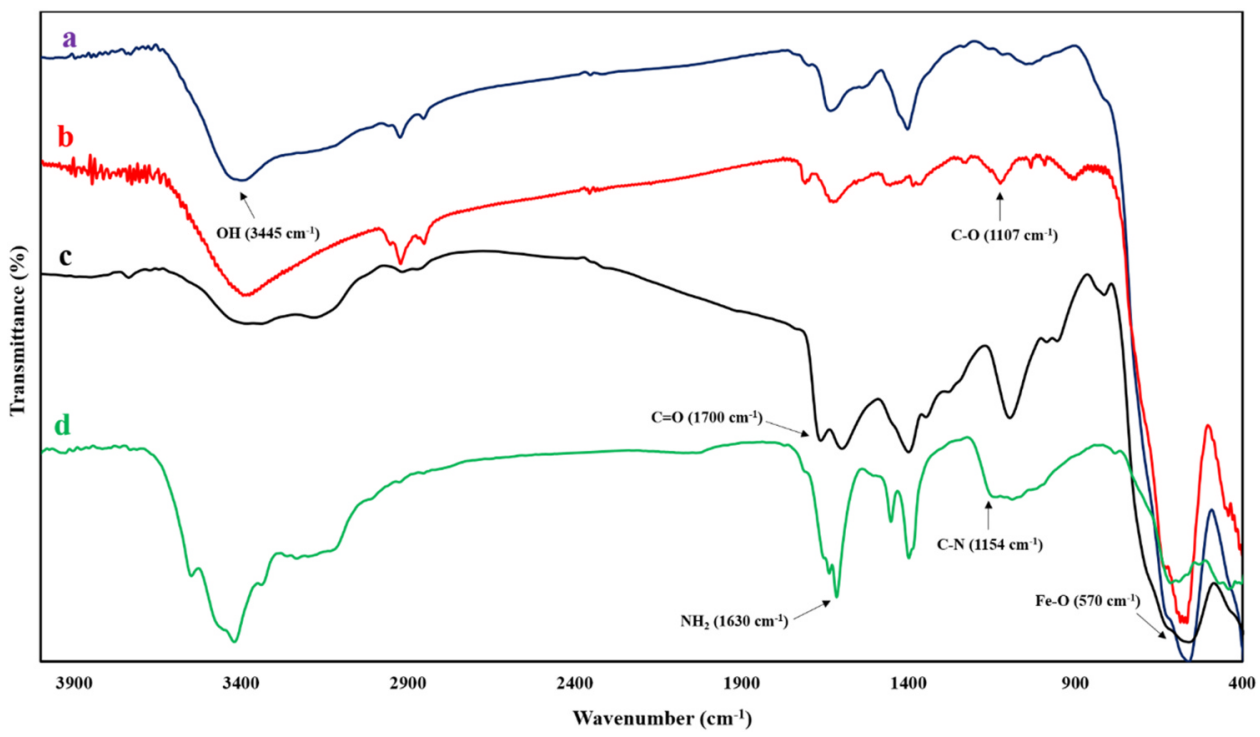

Fig. 4. FT-IR spectra of (a) $\mathrm{Fe}_{3} \mathrm{O}_{4}$, (b) $\mathrm{Fe}_{3} \mathrm{O}_{4} @ P E G$, (c) propanamide-contained DMIP and (d) propanamide-removed DMIP. 
similar shape and non-hysteresis which confirmed they had super paramagnetic behavior. The magnetic saturation of bare $\mathrm{Fe}_{3} \mathrm{O}_{4}\left(66.5\right.$ emug $\left.^{-1}\right)$ was higher than that of DMIP $\left(61.0 \mathrm{emug}^{-1}\right)$, which was related to contribution of PEG and MIP layer that coated $\mathrm{Fe}_{3} \mathrm{O}_{4}$ effectively. It should be noted that magnetic saturation of DMIP was approximately 88 times higher than reported acrylic-based MIP [38], as well as synthesis route was attractive according to green chemistry. In addition, the high magnetic responsivity and hydrophilicity of DMIP facilitated the magnetic isolation of sorbent from sample solution within $5 \mathrm{~s}$ when exposed to external magnetic field; whereas, DMIP was dispersed rapidly with a slight shake when magnetic field didn't exist. As a result, strong magnetism of DMIP caused fast accumulation of sorbent and subsequently shortened sample preparation time significantly.

\subsection{Binding study of the DMIP for acrylamide}

Binding study of the DMIP for acrylamide was performed including adsorption kinetics, static adsorption (binding isotherm) and corresponding models. The adsorption kinetics of acrylamide onto the DMIP and NIP at different time intervals were firstly studied. Fig. S4a reveals that both DMIP and NIP exhibited fast adsorption rate which could be attributed to the thin imprinted-layer leading to substantial accessibility to the specific binding cavities and subsequently fast masstransfer of acrylamide into them. As seen from the binding saturation plot (Fig. S4a), the adsorption capacity increased rapidly in the first $10 \mathrm{~min}$, and then the increment slowed down until the process approximately reached equilibrium after 20 min which is almost 7.5 times shorter than reported acrylic-based surface imprinting MIPs [39]. At the beginning of the adsorption process, acrylamide could enter many empty specific binding sites easily and rapidly and mass-transfer resistance was significantly small. With time prolonging, it became difficult to find an imprinted site for acrylamide. So, the adsorption rate decelerated up to reaching equilibrium. The Pseudo-first order and Pseudo-second order kinetic models were used to investigate the adsorption kinetics. These kinetic models and their corresponding parameters were listed in Table S2. The results indicated that Pseudo-second order kinetic model was more suitable for fitting the kinetic data due to its higher $\mathrm{R}^{2}$ value than Pseudo-first order kinetic model. Then, based on the assumptions of the Pseudo-second order kinetic model, it was assumed that chemical interactions were possibly involved in the adsorption process, which may involve valency forces through sharing or exchange of electrons between acrylamide and DMIP/NIP.

The binding isotherm of analyte onto DMIP and NIP is a notable factor to appraise the recognition capability of imprinting memory and specific binding. Accordingly, static adsorption capacities of DMIP and NIP for acrylamide were examined in different concentrations of acrylamide from 50 to $600 \mathrm{mg} \mathrm{L}^{-1}$. Fig. S4b indicates that the amount of acrylamide adsorbed by both DMIP and NIP increased by raising the initial concentration up to $450 \mathrm{mg} \mathrm{L}^{-1}$, and subsequently binding sites reached saturation. Various isotherm models such as Freundlich, Langmuir, Tempkin and Dubinin-Radushkevich were applied for analyzing binding properties of DMIP and NIP. As shown in Table S3, Freundlich equation with $\mathrm{R}^{2}>0.99$ was a better model to reflect the adsorption behaviors, which supposed that the adsorption referred to heterogeneous surfaces. Therefore, Freundlich isotherm was selected for fitting the isotherm data to estimate binding mechanisms by the below equation [40]:

$\log Q=m \log C+\log \alpha$

where $Q\left(\mathrm{mg} \mathrm{g}^{-1}\right)$ is the binding capacity of the polymers at the equilibrium concentration, $C\left(\mathrm{mg} \mathrm{L}^{-1}\right)$ is equilibrium acrylamide concentration in sample solution, $\alpha$ corresponds to Freundlich constant demonstrating average affinity and measure of capacity while $m$ is heterogeneity index which can take values from 1 to 0 . The excessive $m$ value of 1 reveals that the system is homogeneous and 0 value refers to heterogeneous systems.
Fig. S4c displays the adsorption isotherm for acrylamide on the polymers that were fitted to Freundlich isotherm with linear correlation coefficients 0.993 and 0.955 for DMIP and NIP respectively, which indicated both polymers contained heterogeneous binding sites that was in good agreement with most non-covalently imprinted polymers [41]. The $m$ value for DMIP (0.3805) lower than that for NIP $(0.8527)$ suggested that DMIP had more heterogeneous binding sites than NIP due to a substantial role of template molecule during polymerization. The maximum adsorption capacities achieved for acrylamide were $24.1 \mathrm{mg} \mathrm{g}^{-1}$ by DMIP, much higher than that by NIP $\left(6.9 \mathrm{mg} \mathrm{g}^{-1}\right)$, proving that propanamide was qualified molecule as dummy template and specific cavities were successfully formed in the DMIP structure.

Moreover, to evaluate the affinity of DMIP toward acrylamide, Scatchard analysis was used which can be defined as follows:

$Q / C_{e}=\left(Q_{\max }-Q\right) K_{d}$

where $Q$ is the amount of acrylamide bound to the DMIP at equilibrium, $C_{\mathrm{e}}$ is the equilibrium concentration of acrylamide in solution, $Q_{\max }$ is the apparent maximum binding amount and $K_{\mathrm{d}}$ is the dissociation constant of the binding sites. As shown in Fig. S4d, two different straight lines were obtained for the DMIP Scatchard plot, indicating there were two distinct binding sites: the high-affinity (specific) (left side) and the low-affinity (non-specific) (right side) sites. According to the slopes of these two straight lines, the corresponding dissociation constants $\left(K_{\mathrm{d}}\right)$ values were calculated to be 43.85 and $250 \mathrm{mg} \mathrm{L}^{-1}$ for the high and low affinity binding sites with correlation coefficients $\left(\mathrm{R}^{2}\right)$ of 0.9640 and 0.9277 , respectively. That means corresponding affinity constants were 0.023 and 0.004 , respectively.

\subsection{Selectivity examination of DMIP}

Selectivity of MIPs is the chief and prominent advantage of MIPs. In this regard, the selectivity of DMIP was appraised by comparing adsorption capacity, imprinting factors (IF) and selectivity factors (SF) for acrylamide and other similar compounds including propanamide, $\mathrm{L}$ asparagine, 6-aminocaproic acid and N-tert-Butylacrylamide. As seen in Fig. 5 the adsorption capacity for propanamide is the highest that is owing to remarkable matching among imprinted cavities and propanamide structure in terms of size, stereochemistry and functionality. It should be pointed out that the adsorption capacity for acrylamide is slightly lower than propanamide which can affirm that propanamide is highly qualified molecule as a dummy template for acrylamide. In other word, high symmetry of acrylamide with propanamide caused acrylamide to easily enter to imprinted cavities and no barrier existed for mass-transfer of analyte. However, for other three analogue compounds, significant distinctions in volume, shape and functional-group position decreased the affinity of DMIP toward them. In addition, as shown in Fig. 5, the IF values of DMIP for acrylamide, propanamide, Lasparagine, 6-aminocaproic acid and N-tert-Butylacrylamide were 3.49, $3.54,2.14,1.73$ and 1.5 , respectively, while the SF values were the four analogues were $0.90,1.67,2.13$ and 2.48 , respectively. These results indicated that the propanamide dummy DMIP had highly selective recognition capability toward acrylamide.

\subsection{Variable optimization for DMIP based MSPE}

In order to achieve maximum extraction recovery of acrylamide in biscuit samples, the preliminary effects of main variables including sample solution $\mathrm{pH}$, DMIP dosage, extraction time, kind and volume of desorption solvent were investigated, at the spiked concentration level of $200.0 \mu \mathrm{g} \mathrm{L}^{-1}$.

Acrylamide is an extremely hydrophilic compound that could exist in biscuit samples at the presence of matrix interferences especially long chain fatty acids which could seriously pollute/block HPLC column or give interferent peaks overlapping with peak of acrylamide. To dominate the obstacles, a number of researchers have used large volumes of 

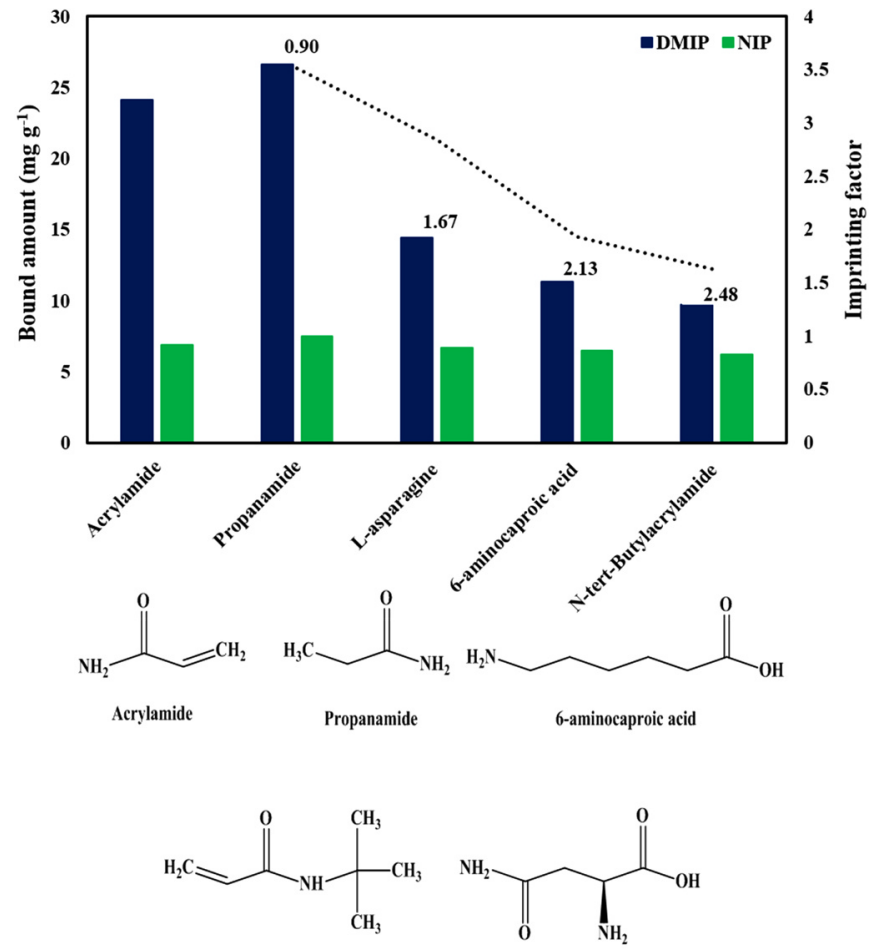

N-tert-Butylacrylamide

L-asparagine

Fig. 5. (Upper) Selectivity of the DMIP and NIP for acrylamide over four analogues including propanamide, L-asparagine, 6-aminocaproic acid and N-tertButylacrylamide; (below) chemical structures of the tested five compounds. Experimental conditions: $30 \mathrm{mg}$ of DMIP or NIP, concentration of acrylamide and four analogues at $400 \mathrm{mg} \mathrm{L}^{-1}$ individually.

toxic organic solvent (hexane) as rinsing solvent to remove hydrophobic impurities due to non-solubility of acrylamide in hexane or used commercial SPE cartridges for clean-up, which usually include several steps and are time/labor consuming $[42,43]$. To eliminate the utilization of organic solvent and waste prevention, herein, we used ice bath for solidification of oil layer which could easily be removed for defating sample solution. Thus, using ice bath could be a superior alternative instead of organic solvent in washing step, which could facilely avoid interferences.

For selecting appropriate polarity of desorption solvent, solubility of the target analyte and its compatibility with chromatographic system should be considered. Due to the good polarity and miscibility of acrylamide, different types of polar solvents with potent rinsing strength were examined, including acetonitrile, methanol, deionized water, methanol/acetonitrile (50:50, v/v), acetonitrile/ammonium hydroxide (90:10, v/v), methanol/ammonium hydroxide (90:10, v/v), and deionized water/ammonium hydroxide (90:10, v/v). Among the tested desorption solvents, methanol/ammonium hydroxide (90:10, v/v) provided maximum desorption efficiency, in which most amounts of acrylamide could be desorbed from the DMIP. This may be due to the similarity between the polarity of acrylamide and methanol and high solubility of acrylamide in methanol which could break hydrogen bonds between trapped analyte and sorbent easily. In addition, the effect of volume of the selected desorption solvent was tested in the range of $1.0-5.0 \mathrm{~mL}$ and results demonstrated the adequacy of $2.0 \mathrm{~mL}$ of the solvent to desorb acrylamide completely.

\subsection{Variable effects evaluation by CCD and RSM}

Conventional optimization approaches namely one-variable-at-atime have been widely used, which often suffer from deficiency viz. necessity too much extents of experimental run, labor effort, high amounts of chemical waste production, time consuming, high cost and most importantly incapable to distinguish the significance of each variable, and ignoring the effects of interaction between variables and subsequently unable to attain the true optimum level. To overcome the mentioned drawbacks, multivariate optimization is a proper and applicable statistical approach that could eliminate the limitations of conventional optimization [44]. Central composite design (CCD) based response surface methodology (RSM) provides contribution of individual variables and their interactions on target response empirically polynomial function at least of expense and time $[45,46]$. Therefore, valuable information for a predestinated process was attained via experimental design methodologies. Hence, in this study the influence of DMIP dosage (A), sonication time (B) and sample solution $\mathrm{pH}(\mathrm{C})$ as three effective main parameters on MSPE of acrylamide from biscuit samples were evaluated via CCD based on RSM as an effective statistical and mathematical approach for assessment and optimization of parameters and their interactions. The total number of design points in CCD was 15 including 4 fractional factorial points, 6 axial points and 5 central points, as listed in Table S4. Among different statistic models including linear, 2FI, quadratic and cubic, quadratic model based analysis of variance (ANOVA) has been widely applied to investigate the statistical significance of factors. The significance and adequacy of model, parameters, their interactions and quadratic effects on extraction process were investigated through ANOVA (Table S5) based on Fisher's tests (acceptance or rejection of the null hypothesis). At certain confidence level $(\alpha=0.05)$, the terms with higher values of $F$ or $p$ values less than 0.05 strongly support their significant contribution on extraction process. The reliability and robustness of fitted quadratic model were checked via lack-of-fit test and multiple correlation coefficients. In this proposed model, the lack-of-fit value higher than 0.05 and the correlation coefficient higher than 0.98 have confirmed the high accuracy and reliability of suggested models. The obtained results from CCD for each parameter were fitted to a polynomial equation with quadratic multiple regressions, which expressed the relationship between response and variables. Extraction recovery (ER) was used as the indicator of MSPE. Accordingly, the second order polynomial equation of acrylamide was generated as below:

$E R \%=+87.44+8.93 A+10.00 B-6.59 C+5.52 A B+14.48 A C+4.27 B C$

$$
-2.91 A^{2}-3.86 B^{2}-4.90 C^{2}
$$

Response surface 3D plots provided valuable information about influential interactions of main parameters, as shown in Fig. S5. By considering the chemical structures of the acrylamide (ionic or natural form) and DMIP, the $\mathrm{pH}$ of the sample solution had significant effect on the dominant sorption mechanism (electrostatic and hydrogen bonding interactions) between the analyte and the sorbent, and therefore impressive influences on extraction efficiency. Fig. S5a depicts semi-curvature plot between sample $\mathrm{pH}$ and DMIP dosage that indicated interactive effects. As seen, by increasing initial sample $\mathrm{pH}$ up to 5 the extraction efficiency was increased, then in $\mathrm{pH} 6$ the efficiency decreased slightly. After pH 6 the extraction efficiency diminished remarkably. As a matter of fact, acrylamide is a sorely weak base, and at $\mathrm{pH}$ lower than 3.0 the protonated form starts to emanate and induces a positive charge to its structure. At any other $\mathrm{pH}$ above 3 acrylamide is in its molecular (natural) form, so no $\mathrm{pH}$ dependency can be found. However, the recorded $\mathrm{pH}$ dependency of the binding efficiency was most probably given by the dissociation process of the chitosan $\mathrm{NH}_{3}$ groups ( $\mathrm{p} K_{\mathrm{a}}$ value $\sim 6.5$ ). Amide group of acrylamide and amine group of chitosan ( $\mathrm{p} K_{\mathrm{a}}$ value $\sim 6.5$ ) were easily protonated concurrently at $\mathrm{pH}$ below 3.0, which established major repulsion leading to deteriorative extraction efficiency. At any other $\mathrm{pH}$ above 3.0, acrylamide would be deprotonated and also would be in molecular form. On the other hand, at acidic $\mathrm{pH}$ (below $\sim 6.5$ ) chitosan gained dominant positive charge (due to $\mathrm{NH}_{2}$ group). As a result, carbonyl group of acrylamide had impressive affinity to link with $\mathrm{NH}_{3}{ }^{+}$group of chitosan by ionic interaction. Furthermore, $\mathrm{NH}_{2}$ group of acrylamide could interact with 
other functional groups of chitosan viz. -O- and - $\mathrm{OH}$ by hydrogen bonding and thereby provide additional driving forces; this would accompany with memory effects built in DMIP structure and thereby would enhance extraction efficiency remarkably. On the other hand, in basic media both acrylamide and chitosan are in molecular dominant species form leading to deteriorative interactions and subsequently poor extraction recovery. As seen in Fig. S5, maximum efficiency was attained at $\mathrm{pH} 5.0$ which was completely in agreement with above claim.

Ultrasound irradiations could provide influential mixing by cavitation phenomenon that would impel excellent dispersion of sorbent in sample solution, resulting in that the mass transfer of analyte to sorbent occurs gradually effectively. Fig. S5c shows the extraction efficiency decreased obviously using low amounts of sorbent and short sonication time, which could be ascribed to the probable formation of DMIP aggregate due to the slight opportunity for analyte to penetrate into binding sites. By simultaneous increment of sorbent dosage and sonication time, the preconcentration of acrylamide was raised gradually, that could be attributed to the increased available specific active sites and surface areas which are qualified for trapping acrylamide molecule along with great dispersion compelled by cavitation phenomenon which universally led to significant betterment of mass transfer. As depicted in Eq. (4), the coefficients of parameter AC were the highest that demonstrated it had the most impact on the extraction efficiency while the positive or negative coefficients of the parameter represented the direction of the effect on the response.

As a result, the maximum extraction recoveries were achieved at acidic $\mathrm{pH}$ of $5.0,30 \mathrm{mg}$ of DMIP and sonication time of $20 \mathrm{~min}$ with desirability 0.90 (the value of 1.0 corresponds to highest extraction percentage and extremely desirable condition; score 0.0 illustrates minimum extraction recovery), which was repeated for three times to investigate applicability of this optimum level. The desirability value for individual parameters was displayed in Fig. S6. According to Fig. S7, the adequate compatibility between experimental and predicted results and desirability certification were proved with high sufficiency of the purposed model.

\subsection{Method validation and real sample analysis}

In order to validate the developed DMIP-MSPE-HPLC/UV method for the determination of acrylamide, further experiments with regard to calibration linearity range, limit of detection (LOD), limit of quantification (LOQ), robustness and accuracy were conducted under the optimized experimental conditions. The calibration curves were built by plotting peak area versus acrylamide concentration in the wide range of $2.0-5000.0 \mu \mathrm{g} \mathrm{kg}^{-1}$ and $5.0-5000.0 \mu \mathrm{g} \mathrm{kg}^{-1}$ with the correlation coefficients of 0.9991 and 0.9985 for standard aqueous solution and biscuit samples, respectively, demonstrating excellent linearity. The LOD and LOQ defined as three times and ten times ratio of signal to noise for chromatograms obtained from the blank [47], respectively, were $0.57 \mu \mathrm{g} \mathrm{kg}^{-1}$ and $1.90 \mu \mathrm{kg}^{-1}$ for standard aqueous solution. For biscuit samples, the LOD and LOQ were 1.3 and $4.4 \mu \mathrm{g} \mathrm{kg}^{-1}$, respectively, which proved worthy operational condition and competency of the present method. Owing to the more complex matrices in biscuit samples compared with standard aqueous solutions, the LOD and LOQ values in biscuit samples were slightly higher. Despite the existence of some published papers using calibration curves in standard aqueous solutions, it is more reasonable and scientific for the method validation in real samples, owing to fully considering the matrix effects and presenting more accurate results. So, we adopted the calibration curves in biscuit samples. The repeatability and reproducibility (intra-day and inter-day precisions) were assessed by determination of acrylamide spiked standard in biscuit samples at five different concentration level $\left(5.0,50.0,200.0,500.0\right.$ and $\left.5000.0 \mu \mathrm{g} \mathrm{kg}^{-1}\right)$. As seen in Table 1 , the extraction recoveries and relative standard deviations (RSDs) of intraday precision were found to be $86.0-98.3 \%$ and $1.2-3.0 \%$, respectively,
Table 1

Intra-day and inter-day precision and recovery of the DMIP-MSPE-HPLC/UV method for acrylamide determination in spiked biscuit samples $(n=4)$.

\begin{tabular}{llll}
\hline Add $\left(\mu \mathrm{kg}^{-1}\right)$ & Found $\left(\mu \mathrm{kg}^{-1}\right) \pm \mathrm{SD}$ & RSD (\%) & Recovery (\%) \\
\hline Intra-day & & & \\
5.0 & $4.3 \pm 0.13$ & 3.0 & 86.0 \\
50.0 & $47.5 \pm 1.30$ & 2.7 & 95.0 \\
200.0 & $187.5 \pm 3.32$ & 1.8 & 93.8 \\
500.0 & $456.0 \pm 12.54$ & 2.8 & 91.2 \\
5000.0 & $4915.0 \pm 59.72$ & 1.2 & 98.3 \\
Inter-day & & & \\
5.0 & $4.4 \pm 0.18$ & 4.1 & 88.0 \\
50.0 & $43.5 \pm 1.30$ & 3.0 & 87.0 \\
200.0 & $182.0 \pm 2.82$ & 1.6 & 91.0 \\
500.0 & $488.0 \pm 12.75$ & 2.6 & 97.6 \\
5000.0 & $4870.0 \pm 87.18$ & 1.8 & 97.4 \\
\hline
\end{tabular}

while that of inter-day recoveries and precisions were $87.0-97.6 \%$ and $1.6-4.1 \%$, respectively, which indicated excellent reproducibility of the developed method. Meanwhile, the accuracy and applicability of the DMIP-MSPE-HPLC/UV method were validated for extraction and preconcentration of acrylamide in biscuit samples.

Fig. 6 shows the chromatograms of biscuit samples. As shown in Fig. 6a, the chromatogram of direct injection of biscuit without applying DMIP-MSPE was very complex, and due to the overlapping of interference peaks with the peak of acrylamide, acrylamide couldn't be found. Even though the $y$-scale of chromatogram changed to 0-15 $\mathrm{mAu}$, the acrylamide's peak wasn't seen yet (inset of Fig. 6a). After spiking the standard solution of acrylamide, the direct injection of biscuit sample without pretreatment step revealed that matrix impurities seriously overlapped with acrylamide's peak (Fig. 6b), so quantitative analysis of acrylamide was still impossible. Interestingly, after DMIP-MSPE, most of impurity signals of the biscuit samples were greatly reduced (Fig. 6c and d), owing to high clean-up potential of the DMIP-MSPE. More excitingly, for the spiked biscuit sample, the interference-free acrylamide's peak emerged significantly (Fig. 6d), proving high potential of the DMIP-MSPE method for clean extraction of acrylamide from complicated biscuit samples. Consequently, the DMIP-MSPE method was practically applicable.

Furthermore, five kinds of different biscuit samples were analyzed. As listed in Table 2, the acrylamide was detected in all biscuit samples within $65.8-2962.4 \mu \mathrm{g} \mathrm{kg}^{-1}$ and the RSDs values were lower than $3.3 \%$ for three replicated extractions, indicating the high accuracy of the developed method. These results proved that the established DMIPMSPE method was capable for influential clean-up and preconcentration of acrylamide from the complicated food samples with high precision and accuracy.

\subsection{Reusability of the DMIP}

Reusability of a sorbent is the major profit in terms of practical applications and economic cost. Consequently, several adsorption-desorption cycles of DMIP were performed and results showed that five sequential recoveries were attained of $96.6 \%, 94.1 \%, 91.9 \%, 88.5 \%$, and $86.2 \%$, respectively. As can be seen from Fig. S8, after at least five times, the extraction recovery didn't decrease significantly. Thus, the DMIP-MSPE based method benefited from the advantages of cost-effectiveness, durability, rapidity and simplicity, and could highly sensitive and accurately quantify acrylamide in complicated aqueous samples.

\subsection{Method performance comparison}

The analytical characteristics of the developed DMIP-MSPE-HPLC/ UV method was compared with previously reported literatures for the determination of acrylamide in various sample matrices, as listed in Table S6 [15,31,32,43,48-56]. As can be seen, our achieved LOD and 

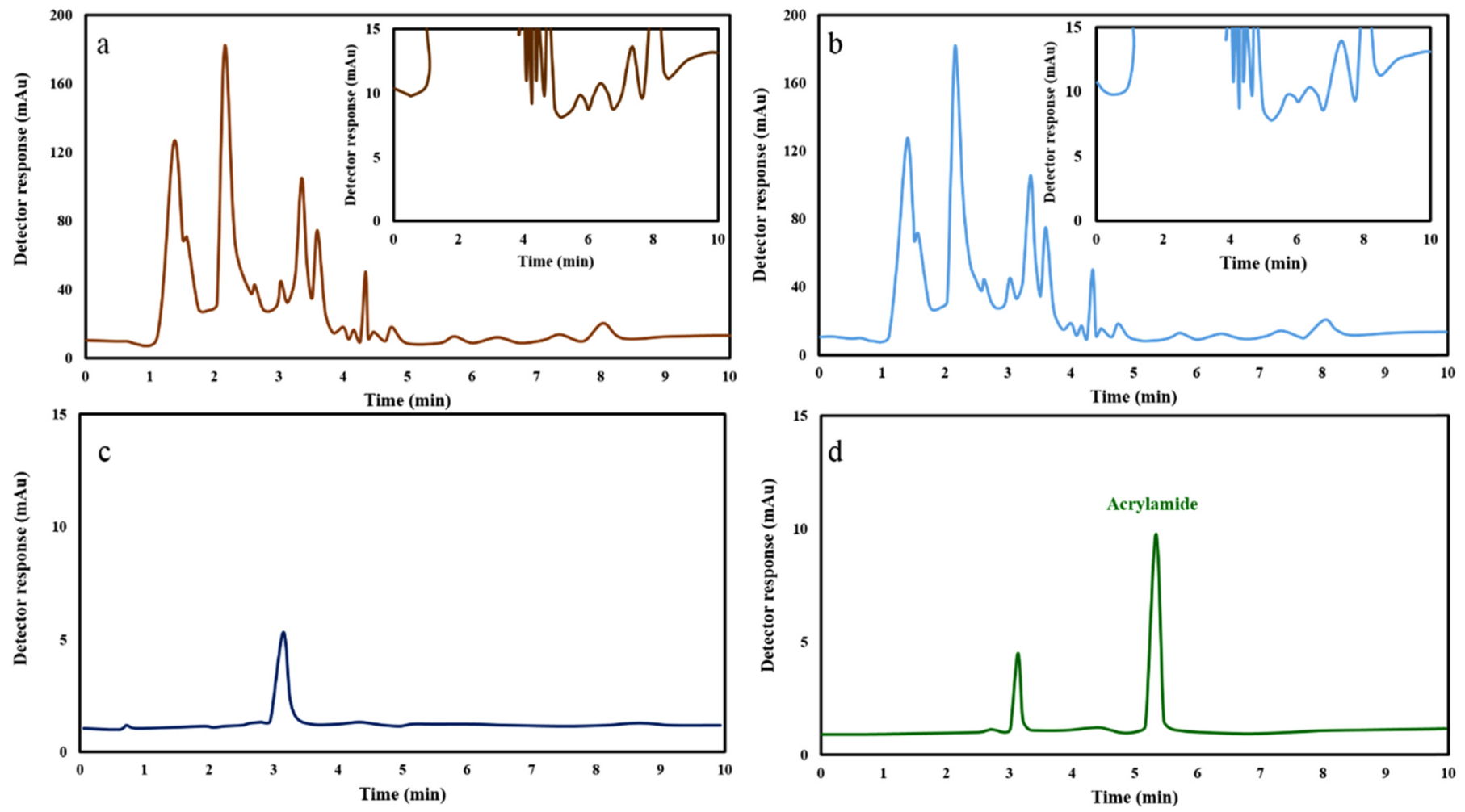

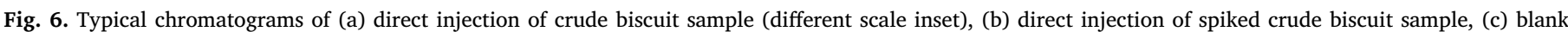
biscuit sample after DMIP-MSPE procedure and (d) spiked biscuit sample after DMIP-MSPE procedure. Spiked concentration: $10 \mu \mathrm{g} \mathrm{kg}^{-1}$.

Table 2

Endogenous contents of acrylamide in five different biscuit samples determined by DMIP-MSPE-HPLC/UV method $(n=3)$.

\begin{tabular}{lll}
\hline Sample & Found $\left(\mu \mathrm{kg}^{-1}\right) \pm \mathrm{SD}$ & $\mathrm{RSD}(\%)$ \\
\hline Biscuit 1 & $270.1 \pm 7.1$ & 2.6 \\
Biscuit 2 & $2962.4 \pm 41.0$ & 1.4 \\
Biscuit 3 & $955.1 \pm 14.8$ & 1.5 \\
Biscuit 4 & $524.7 \pm 11.6$ & 2.2 \\
Biscuit 5 & $65.8 \pm 2.2$ & 3.3
\end{tabular}

linear range is similar to other researches [49,54]. More excitingly, the LOD is also quite low in comparison with some fluorimetry and MSbased detection methods [43,50-53], which is very likely owing to the highly selective preconcentration ability of the DMIP-MSPE. Emphasis should be put on in the current work for the first time both synthesis and extraction procedure were in the line of sustainable and green chemistry with at least consumption of organic solvents and reagents in mild condition which is unique in terms of feasibility, natural origin, full biodegradability, and eco-friendliness for determination of acrylamide.

\section{Conclusions}

In summary, a novel water-compatible DMIP with superparamagnetic behavior was rationally fabricated according to clean and low-cost strategy at least of time by combining dummy and surface imprinting technology with natural monomer. The DMIP was employed as versatile sorbent at the service of MSPE for clean-up and preconcentration of acrylamide from biscuit samples. During MSPE, washing step was intelligently omitted while long chain fatty acids impurities were removed by using ice bath which was an excellent alternative to toxic organic solvent (e.g., hexane). Both synthesis route and extraction procedure benefited from the impressive advantages, like time saving, waste eliminating, environmentally benign substances and minimum chemical pollutions, minimum amount of glassware and working at mild condition. By immobilizing thin layer imprinted chitosan network onto $\mathrm{Fe}_{3} \mathrm{O}_{4}$ @PEG core, the DMIP provided fast equilibrium kinetics and high adsorption capacity, which decreased analysis time and increased aqueous applicability. The versatile DMIP not only denoted high magnetization that could be quickly separated from the suspension by magnetic field without requirement to SPE column packing, filtration, or centrifugation, but also the interference of the leaching residual template was completely circumvented by dummy imprinting strategy. Furthermore, by using multivariable optimization based on experimental design methodology, impacts of individual parameters and their interactions on the extraction efficiency were studied and subsequently the true optimum level was found with the minimum of expense, time, labor effort and chemicals. Consequently, we believe these appropriate features could contribute to enhancing the performances of MIPs in line of clean chemistry especially biopolymer usage, and thereby promote the rapid development and wide applications of molecular imprinting.

\section{Acknowledgments}

This work was financially supported by Graduate School and Research Council of Yasouj University and the National Natural Science Foundation of China $(21876199,21804010,41776110)$, and the Department of Science and Technology of Shandong Province of China (GG201709290055).

\section{Appendix A. Supporting information}

Supplementary data associated with this article can be found in the online version at doi:10.1016/j.talanta.2018.11.065. 


\section{References}

[1] IARC, Acrylamide. Monographs on the Evaluation Of Carcinogenic Risks To Humans: Some Industrial Chemicals, 60, 1994, pp. 389-433.

[2] E. Tareke, P. Rydberg, P. Karlsson, S. Eriksson, M. Törnqvist, Analysis of acrylamide, a carcinogen formed in heated foodstuffs, J. Agric. Food Chem. 50 (2002) 4998-5006.

[3] D.S. Mottram, B.L. Wedzicha, A.T. Dodson, Food chemistry: acrylamide is formed in the Maillard reaction, Nature 419 (2002) 448-449.

[4] M. Friedman, Chemistry, biochemistry, and safety of acrylamide. A review, J. Agric. Food Chem. 51 (2003) 4504-4526.

[5] S.N.F. administration, Information about acrylamide in food. 〈http://www. konsumentverket.se/html-sidor/livsmedelsverket/engakrylpressmeddelande.htm〉.

[6] M. Lambert, C. Inthavong, F. Hommet, J.-C. Leblanc, M. Hulin, T. Guérin, Levels of acrylamide in foods included in 'the first French total diet study on infants and toddlers', Food Chem. 240 (2018) 997-1004.

[7] C. Cagliero, T.D. Ho, C. Zhang, C. Bicchi, J.L. Anderson, Determination of acrylamide in brewed coffee and coffee powder using polymeric ionic liquid-based sorbent coatings in solid-phase microextraction coupled to gas chromatography-mass spectrometry, J. Chromatogr. A 1449 (2016) 2-7.

[8] M.V. Russo, P. Avino, A. Centola, I. Notardonato, G. Cinelli, Rapid and simple determination of acrylamide in conventional cereal-based foods and potato chips through conversion to 3-[bis (trifluoroethanoyl) amino]-3-oxopropyl trifluoroacetate by gas chromatography coupled with electron capture and ion trap mass spectrometry detectors, Food Chem. 146 (2014) 204-211.

[9] E.L. De Paola, G. Montevecchi, F. Masino, D. Garbini, M. Barbanera, A. Antonelli, Determination of acrylamide in dried fruits and edible seeds using QuEChERS extraction and LC separation with MS detection, Food Chem. 217 (2017) 191-195.

[10] K. Mastovska, S.J. Lehotay, Rapid sample preparation method for LC - MS/MS or GC - MS analysis of acrylamide in various food matrices, J. Agric. Food Chem. 54 (2006) 7001-7008.

[11] A. Ostovan, M. Ghaedi, M. Arabi, Q. Yang, J. Li, L. Chen, Hydrophilic multi-template molecularly imprinted biopolymers based on a green synthesis strategy for determination of B-family vitamins, ACS Appl. Mater. Interfaces 10 (2018) 4140-4150.

[12] X. Wu, X. Wang, W. Lu, X. Wang, J. Li, H. You, H. Xiong, L. Chen, Water-compatible temperature and magnetic dual-responsive molecularly imprinted polymers for recognition and extraction of bisphenol A, J. Chromatogr. A 1435 (2016) 30-38.

[13] S. Ansari, Application of magnetic molecularly imprinted polymer as a versatile and highly selective tool in food and environmental analysis: recent developments and trends, TrAC, Trends Anal. Chem. 90 (2017) 89-106.

[14] L. Chen, X. Wang, W. Lu, X. Wu, J. Li, Molecular imprinting: perspectives and applications, Chem. Soc. Rev. 45 (2016) 2137-2211.

[15] F. Ning, T. Qiu, Q. Wang, H. Peng, Y. Li, X. Wu, Z. Zhang, L. Chen, H. Xiong, Dummy-surface molecularly imprinted polymers on magnetic graphene oxide for rapid and selective quantification of acrylamide in heat-processed (including fried) foods, Food Chem. 221 (2017) 1797-1804.

[16] S. Xu, H. Lu, J. Li, X. Song, A. Wang, L. Chen, S. Han, Dummy molecularly imprinted polymers-capped CdTe quantum dots for the fluorescent sensing of 2, 4, 6-trinitrotoluene, ACS Appl. Mater. Interfaces 5 (2013) 8146-8154.

[17] J. Qiao, M. Wang, H. Yan, G. Yang, Dispersive solid-phase extraction based on magnetic dummy molecularly imprinted microspheres for selective screening of phthalates in plastic bottled beverages, J. Agric. Food Chem. 62 (2014) 2782-2789.

[18] H. Zhang, Water-compatible molecularly imprinted polymers: promising synthetic substitutes for biological receptors, Polymer 55 (2014) 699-714.

[19] M. Arabi, M. Ghaedi, A. Ostovan, Water compatible molecularly imprinted nanoparticles as a restricted access material for extraction of hippuric acid, a biological indicator of toluene exposure, from human urine, Microchim. Acta 184 (2017) 879-887.

[20] Y. Liu, L. Zhang, N. Zhao, Y. Han, F. Zhao, Z. Peng, Y. Li, Preparation of molecularly imprinted polymeric microspheres based on distillation-precipitation polymerization for an ultrasensitive electrochemical sensor, Analyst 142 (2017) 1091-1098.

[21] Y. Yang, G. Fang, G. Liu, M. Pan, X. Wang, L. Kong, X. He, S. Wang, Electrochemical sensor based on molecularly imprinted polymer film via sol-gel technology and multi-walled carbon nanotubes-chitosan functional layer for sensitive determination of quinoxaline-2-carboxylic acid, Biosens. Bioelectron. 47 (2013) 475-481.

[22] J. Pan, L. Li, H. Hang, R. Wu, X. Dai, W. Shi, Y. Yan, Fabrication and evaluation of magnetic/hollow double-shelled imprinted sorbents formed by Pickering emulsion polymerization, Langmuir 29 (2013) 8170-8178.

[23] M. Arabi, M. Ghaedi, A. Ostovan, Development of a lower toxic approach based on green synthesis of water-compatible molecularly imprinted nanoparticles for the extraction of hydrochlorothiazide from human urine, ACS Sustain. Chem. Eng. 5 (2017) 3775-3785.

[24] Q. You, Y. Zhang, Q. Zhang, J. Guo, W. Huang, S. Shi, X. Chen, High-capacity thermo-responsive magnetic molecularly imprinted polymers for selective extraction of curcuminoids, J. Chromatogr. A 1354 (2014) 1-8.

[25] E. Moczko, A. Guerreiro, E. Piletska, S. Piletsky, PEG-stabilized core-shell surfaceimprinted nanoparticles, Langmuir 29 (2013) 9891-9896.

[26] A. Durdureanu-Angheluta, M. Pinteala, B.C. Simionescu, Tailored and functionalized magnetite particles for biomedical and industrial applications, Materials Science and Technology, (2012), pp. 149-178 (InTech).
[27] E. Amstad, T. Gillich, I. Bilecka, M. Textor, E. Reimhult, Ultrastable iron oxide nanoparticle colloidal suspensions using dispersants with catechol-derived anchor groups, Nano Lett. 9 (2009) 4042-4048.

[28] J. Sánchez-González, M.J. Tabernero, A.M. Bermejo, P. Bermejo-Barrera, A. Moreda-Piñeiro, Development of magnetic molecularly imprinted polymers for solid phase extraction of cocaine and metabolites in urine before high performance liquid chromatography-tandem mass spectrometry, Talanta 147 (2016) 641-649.

[29] Z. Cui, Y. Xiang, J. Si, M. Yang, Q. Zhang, T. Zhang, Ionic interactions between sulfuric acid and chitosan membranes, Carbohydr. Polym. 73 (2008) 111-116.

[30] X. Liu, L.G. Mao, Y.L. Wang, X.B. Shi, Y. Liu, Y. Yang, Z. He, Electrochemical sensor based on imprinted sol-gel polymer on Au NPs-MWCNTs-CS modified electrode for the determination of acrylamide, Food Anal. Method. 9 (2016) 114-121.

[31] L. Xu, X. Qiao, Y. Ma, X. Zhang, Z. Xu, Preparation of a hydrophilic molecularly imprinted polymer and its application in solid-phase extraction to determine of trace acrylamide in foods coupled with high-performance liquid chromatography, Food Anal. Method. 6 (2013) 838-844.

[32] M. Arabi, M. Ghaedi, A. Ostovan, Development of dummy molecularly imprinted based on functionalized silica nanoparticles for determination of acrylamide in processed food by matrix solid phase dispersion, Food Chem. 210 (2016) 78-84.

[33] M. Arabi, A. Ostovan, M. Ghaedi, M.K. Purkait, Novel strategy for synthesis of magnetic dummy molecularly imprinted nanoparticles based on functionalized silica as an efficient sorbent for the determination of acrylamide in potato chips: optimization by experimental design methodology, Talanta 154 (2016) 526-532.

[34] Y. Liu, X. Hu, L. Bai, Y. Jiang, J. Qiu, M. Meng, Z. Liu, L. Ni, A molecularly imprinted polymer placed on the surface of graphene oxide and doped with Mn (II)doped $\mathrm{ZnS}$ quantum dots for selective fluorometric determination of acrylamide, Microchim. Acta 185 (2018) 48-55.

[35] L. Zang, J. Qiu, X. Wu, W. Zhang, E. Sakai, Y. Wei, Preparation of magnetic chitosan nanoparticles as support for cellulase immobilization, Ind. Eng. Chem. Res. 53 (2014) 3448-3454.

[36] W. Xie, J. Wang, Immobilized lipase on magnetic chitosan microspheres for transesterification of soybean oil, Biomass Bioenergy 36 (2012) 373-380.

[37] C.H. Kuo, Y.C. Liu, C.M.J. Chang, J.H. Chen, C. Chang, C.J. Shieh, Optimum conditions for lipase immobilization on chitosan-coated $\mathrm{Fe}_{3} \mathrm{O}_{4}$ nanoparticles, Carbohydr. Polym. 87 (2012) 2538-2545.

[38] M. Ding, X. Wu, L. Yuan, S. Wang, Y. Li, R. Wang, T. Wen, S. Du, X. Zhou, Synthesis of core-shell magnetic molecularly imprinted polymers and detection of sildenafil and vardenafil in herbal dietary supplements, J. Hazard. Mater. 191 (2011) 177-183.

[39] A. Mehdinia, T.B. Kayyal, A. Jabbari, M. Ovais, Aziz-Zanjani, E. Ziaei, Magnetic molecularly imprinted nanoparticles based on grafting polymerization for selective detection of 4-nitrophenol in aqueous samples, J. Chromatogr. A 1283 (2013) $82-88$.

[40] H. Freundlich, Über die adsorption in lösungen, Z. für Phys. Chem. 57 (1907) $385-470$.

[41] A.M. Rampey, R.J. Umpleby, G.T. Rushton, J.C. Iseman, R.N. Shah, K.D. Shimizu, Characterization of the imprint effect and the influence of imprinting conditions on affinity, capacity, and heterogeneity in molecularly imprinted polymers using the Freundlich isotherm-affinity distribution analysis, Anal. Chem. 76 (2004) 1123-1133.

[42] E.K. Paleologos, M.G. Kontominas, Determination of acrylamide and methacrylamide by normal phase high performance liquid chromatography and UV detection, J. Chromatogr. A 1077 (2005) 128-135.

[43] W. Zhang, Z. Deng, W. Zhao, L. Guo, W. Tang, H. Du, L. Lin, Q. Jiang, A. Yu, L. He, Determination of trace acrylamide in starchy foodstuffs by HPLC using a novel mixed-mode functionalized calixarene sorbent for solid-phase extraction cleanup, $\mathrm{J}$ Agric. Food Chem. 62 (2014) 6100-6107.

[44] J. Ma, L. Jiang, G. Wu, Y. Xia, W. Lu, J. Li, L. Chen, Determination of six sulfonylurea herbicides in environmental water samples by magnetic solid-phase extraction using multi-walled carbon nanotubes as adsorbents coupled with highperformance liquid chromatography, J. Chromatogr. A 1466 (2016) 12-20.

[45] A. Ostovan, M. Ghaedi, M. Arabi, A. Asfaram, Hollow porous molecularly imprinted polymer for highly selective clean-up followed by influential preconcentration of ultra-trace glibenclamide from bio-fluid, J. Chromatogr. A 1520 (2017) 65-74.

[46] A. Ostovan, M. Ghaedi, M. Arabi, Fabrication of water-compatible superparamagnetic molecularly imprinted biopolymer for clean separation of baclofen from bio-fluid samples: a mild and green approach, Talanta 179 (2018) 760-768.

[47] A.M. Committee, Recommendations for the definition, estimation and use of the detection limit, Analyst 112 (1987) 199-204.

[48] C. Liu, F. Luo, D. Chen, B. Qiu, X. Tang, H. Ke, X. Chen, Fluorescence determination of acrylamide in heat-processed foods, Talanta 123 (2014) 95-100.

[49] D.A. El-Hady, H.M. Albishri, Simultaneous determination of acrylamide, asparagine and glucose in food using short chain methyl imidazolium ionic liquid based ultrasonic assisted extraction coupled with analyte focusing by ionic liquid micelle collapse capillary electrophoresis, Food Chem. 188 (2015) 551-558.

[50] M. Surma, A. Sadowska-Rociek, E. Cieślik, K. Sznajder-Katarzyńska, Optimization of QuEChERS sample preparation method for acrylamide level determination in coffee and coffee substitutes, Microchem. J. 131 (2017) 98-102.

[51] A.R. Ghiasvand, S. Hajipour, Direct determination of acrylamide in potato chips by using headspace solid-phase microextraction coupled with gas chromatographyflame ionization detection, Talanta 146 (2016) 417-422.

[52] L. Molina-Garcia, C.S.P. Santos, A. Melo, J.O. Fernandes, S.C. Cunha, S. Casal, 
Acrylamide in chips and french fries: a novel and simple method using Xanthydrol for Its GC-MS determination, Food Anal. Method 8 (2014) 1436-1445.

[53] L. Luo, Y. Ren, J. Liu, X. Wen, Investigation of a rapid and sensitive non-aqueous reaction system for the determination of acrylamide in processed foods by gas chromatography-mass spectrometry, Anal. Methods 8 (2016) 5970-5977.

[54] S. Huang, S. Lu, C. Huang, J. Sheng, L. Zhang, W. Su, Q. Xiao, An electrochemical biosensor based on single-stranded DNA modified gold electrode for acrylamide determination, Sens. Actuators B Chem. 224 (2016) 22-30.

[55] Y. Zhang, Y. Dong, Y. Ren, Y. Zhang, Rapid determination of acrylamide contaminant in conventional fried foods by gas chromatography with electron capture detector, J. Chromatogr. A 1116 (2006) 209-216.

[56] M.R. Lee, L.Y. Chang, J. Dou, Determination of acrylamide in food by solid-phase microextraction coupled to gas chromatography-positive chemical ionization tandem mass spectrometry, Anal. Chim. Acta 582 (2007) 19-23. 